\title{
Properties of N-Type GaN Thin Film with Si-Ti Codoping on a Glass Substrate
}

\author{
Wei-Sheng Liu ${ }^{1} * \mathbb{\infty}$, Yu-Lin Chang ${ }^{1}{ }^{\mathbb{D}}$, Chun-Yuan Tan ${ }^{1}$, Cheng-Ting Tsai ${ }^{1}$ \\ and Hsing-Chun Kuo 2,3,* \\ 1 Department of Electrical Engineering, Yuan Ze University, Chung-Li 32003, Taiwan; \\ chang.hbt@gmail.com (Y.-L.C.); jimmy36542@gmail.com (C.-Y.T.); kkhandsome861110@gmail.com (C.-T.T.) \\ 2 Department of Nursing, Division of Basic Medical Sciences, Chang Gung University of Science \\ and Technology, Chiayi 61363, Taiwan \\ 3 Research Fellow, Chang Gung Memorial Hospital, Chiayi 61363, Taiwan \\ * Correspondence: wsliu@saturn.yzu.edu.tw (W.-S.L.); kuohc@gw.cgust.edu.tw (H.-C.K.)
}

Received: 16 June 2020; Accepted: 1 July 2020; Published: 5 July 2020

check for updates

\begin{abstract}
In this study, n-type gallium nitride (GaN) films were fabricated by a silicon-titanium (Si-Ti) codoping sputtering technique with a zinc oxide $(\mathrm{ZnO})$ buffer layer on amorphous glass substrates with different post-growth annealing temperatures for optimizing the GaN crystal quality. Si-Ti-codoped n-type GaN films that were thermally annealed at $400{ }^{\circ} \mathrm{C}$ had a low thin-film resistivity of $2.6 \times 10^{-1} \Omega$-cm and a high electron concentration of $6.65 \times 10^{19} \mathrm{~cm}^{-3}$, as determined through Hall measurement. X-ray diffraction (XRD) results revealed a high (002) XRD intensity with a narrow spectral line and a full width at half maximum (FWHM) value that indicated the superior crystal growth of a hexagonal structure of the GaN thin films. In addition, photoluminescence measurement results demonstrated a near-band-edge emission at $365 \mathrm{~nm}$, indicating the crystal growth of GaN thin films on glass substrates. The Burstein-Moss effect was observed in the Tauc plot results, indicating that the Fermi level inside the conduction band moves upward and thus improves the n-type properties of the GaN thin film. X-ray photoelectron spectroscopy measurement results revealed that all atoms doped into the GaN film are present and that both $\mathrm{Si}$ and $\mathrm{Ti}$ atoms bond with $\mathrm{N}$ atoms.
\end{abstract}

Keywords: GaN; Si doping; Ti doping; glass substrate; ZnO buffer layer

\section{Introduction}

In recent decades, semiconductor materials based on III-V compounds are useful or even essential for many commercial technologies as well as for cutting-edge electronic and optoelectronic devices such as high-electron-mobility transistors, heterostructure bipolar transistors, diode lasers, and light-emitting diodes (LEDs) [1]. However, conventional III-V semiconductor materials based on As, $\mathrm{P}$, and $\mathrm{Sb}$, all of which have narrow energy bandgaps, are unsuitable for use in short-wavelength optoelectronic devices in the violet (380-450 nm) and blue (450-475 nm) regions [2]. Among various material systems, III-V nitride-related compound materials are particularly suitable for application in optoelectronic devices with large energy bandgaps. Furthermore, the direct energy bandgaps of nitride-related materials can be widely modulated by incorporating group-III elements to form Indium Nitride ( $\mathrm{InN}, \mathrm{E}_{\mathrm{g}}=0.7 \mathrm{eV}$ ) and Aluminum Nitride (AlN, $\mathrm{E}_{\mathrm{g}}=6.2 \mathrm{eV}$ ). For the versatile designs on the optoelectronic devices, the narrow and wide band-gap organic materials are also explored and could be combined with the nitride-related materials forming the advantageous applications with superior device performances $[3,4]$. 
Among nitride-related materials, gallium nitride $(\mathrm{GaN})$ is a potential candidate for short-wavelength optoelectronic devices, such as LEDs and laser diodes. GaN, with a wurtzite structure, is a III-nitride semiconductor with a wide direct bandgap of $3.39 \mathrm{eV}$, a high melting point, high thermal conductivity, and a high carrier mobility [5]. Moreover, GaN devices have a high impedance, a high breakdown voltage, and a low capacitance. Therefore, GaN-related material systems are suitable for use as high-electron-mobility transistors with a high breakdown voltage in 5G applications [6].

Several deposition methods are available for growing $\mathrm{GaN}$ thin films for fabricating GaN materials, including metalorganic chemical vapor deposition (MOCVD) [7], hydride vapor phase epitaxy (HVPE) [8], and molecular beam deposition (MBE) [9]. These thin-film deposition methods use sapphire substrates for growing high-quality GaN films. However, the thin-film deposition techniques used for GaN growth by MOCVD, MBE, and HVPE are typically accompanied by manufacturing issues such as high fabrication costs, complicated deposition systems, and a need for expensive sapphire substrates. Large-diameter sapphire substrates are not readily available, and their high manufacturing cost limits the applicability of GaN-related optoelectronic devices fabricated on sapphire substrates [10]. To address these issues and fabricate low-cost GaN-based optoelectronic devices, the need to develop new growth methods of GaN films with a low fabrication cost and high throughput is urgent. Magnetron sputtering has recently been studied for high-quality GaN thin-film deposition on glass substrates at low growth temperatures [11]. The advantages of using magnetron sputtering to deposit $\mathrm{GaN}$ thin films on glass substrates include large-scale fabrication with uniform thin-film thickness at a high deposition rate and a low deposition temperature. Moreover, $\mathrm{GaN}$ thin-film growth by magnetron sputtering can be achieved without using toxic metalorganic precursors and with reduced harmful byproducts [11,12].

Although GaN thin films can be deposited by magnetron sputtering, increasing the deposition rate and preventing harmful charging behavior on the surface of the sputtering target to meet the industry fabrication requirements of high productivity and reduced fabrication cost are necessary. In recent decades, pulsed direct current (DC) magnetron sputtering with fewer harmful arc events at the target surface has been introduced as a new deposition technique because it can achieve a higher thin-film deposition rate than conventional Radio-Frequency (RF) magnetron sputtering, as indicated by the results of Jonsson et al. [13]. Consequently, because of the abovementioned advantages, we choose a pulsed-DC sputtering system as a deposition system to reduce harmful byproducts and to deposit uniform GaN thin films on glass substrates with an increased thin-film deposition rate and reduced manufacturing costs at low growth temperatures. An intrinsic GaN film exhibits semi-insulating characteristics because of its wide energy bandgap. For the optoelectronic applications, it is necessary to modulate the carrier concentration and reduce the resistivity of the GaN thin film. To fabricate GaN thin films with higher conductivity, $\mathrm{Si}, \mathrm{Se}, \mathrm{Ge}$, and Ti are often used as n-type dopants.

GaN films doped with Si have been found to exhibit donor states with shallow ionization energy of approximately $30 \mathrm{meV}$ [14]. Additionally, the calculation of density functional theory of first principle revealed that the lattice constants of GaN thin films would be altered slightly by Si doping [15]. The compressive strain would be decreased as the increase in the Si doping in the GaN film. Moreover, carrier concentration and surface flatness can both be improved by Si doping in GaN films [16]. Thus, fewer crystal defects existed in the Si-doped GaN films. In addition, the electron mobility can be increased due to the screening of the scattering effect of crystal defects by Si doping in the GaN film. Furthermore, the compensation of deep energy states was observed to improve the crystal quality of n-GaN films resulting from an increased Si doping concentration [17]. Therefore, Si doping can significantly improve the electrical properties of the GaN film. Thus, a Si target was adopted in the magnetron sputtering system used in this study for the thin-film deposition of GaN. To further improve n-GaN films' conductivity, Ti was also used as a donor dopant in the Si-doped GaN film to form an innovative silicon-titanium (Si-Ti)-codoped GaN film. According to reported studies, the Fermi energy level of the Ti-doped GaN is an order higher than that of undoped GaN thin film, 
which will lead to the increased electron concentration as well as the improvement of the n-type characteristic [18].

Additionally, according to the formation energy calculation from Victor et al., the Ti dopant was located at the surface layer of Ti-doped GaN, which will result in the tendency of Ti atom to replace

$\mathrm{Ga}$ atom for the formation of the Titanium Nitride (TiN) compound [19]. Since the work function of TiN and the lattice constant of Ti doping was similar to that of $\mathrm{GaN}$, and the energy bandgap of TiN was narrower than that of GaN films, it was observed that Ti doping in GaN films can cause a reduction in thin-film resistivity and an increase in the carrier mobility $[20,21]$. For the lattice structure in Ti-doped GaN films, the Ga atoms are easily substituted by Ti atoms in the GaN film because of the low substitution energy of Ti doping without significant lattice distortion [22]. Therefore, the Ti doping was employed for the enhancement of the electrical characteristics of $\mathrm{n}-\mathrm{GaN}$ films. Considering the abovementioned advantages of $\mathrm{Si}$ and Ti doping and their role in improving the thin-film electrical properties, n-type Si-Ti-codoped GaN thin films were deposited on glass substrates. The GaN films in this study were subjected to thermal annealing at room temperature (RT), $200{ }^{\circ} \mathrm{C}$, and $400{ }^{\circ} \mathrm{C}$ to enhance their electrical properties.

\section{Materials and Methods}

In this study, Si-Ti-codoped n-GaN films were deposited on glass substrates with a zinc oxide $(\mathrm{ZnO})$ layer as the buffer layer using a pulsed-DC sputtering system. First, the sputtering chamber was evacuated to a high vacuum of approximately $5.33 \times 10^{-4} \mathrm{~Pa}$. Next, the $\mathrm{ZnO}$ buffer layer was deposited with a thickness of $100 \mathrm{~nm}$ by RF magnetron sputtering; the processing pressure was $1.33 \times 10^{-1} \mathrm{~Pa}$ at a deposition power of $150 \mathrm{~W}$ for a 3-inch-diameter $\mathrm{ZnO}$ target (purity: $99.99 \%$ ), and the argon (Ar) flow rate was $30 \mathrm{sccm}$. After the deposition of the $\mathrm{ZnO}$ buffer layer, the n-GaN film was grown with the thickness of $300 \mathrm{~nm}$ by using the pulsed-DC sputtering technique at deposition power of $75 \mathrm{~W}$ for a 3-inch GaN target (purity: 99.95\%), RF sputtering at $30 \mathrm{~W}$ for a 3-inch Si target, and DC sputtering at $20 \mathrm{~W}$ for a 3-inch Ti target ( $\mathrm{Si}$ and Ti target purity: 99.99\%). GaN, $\mathrm{Si}$, and $\mathrm{Ti}$ were sputter-deposited simultaneously; the processing pressure in the chamber and Ar flow rate were $1.99 \mathrm{~Pa}$ and $15 \mathrm{sccm}$, respectively. Before GaN and $\mathrm{ZnO}$ deposition, the targets were presputtered for 1 min to clean them. The Ar: N2 gas mix ratio was set at 1:1 for growing the $\mathrm{n}-\mathrm{GaN}$ film, and pure Ar gas $(30 \mathrm{sccm})$ was used for depositing the $\mathrm{ZnO}$ buffer layer. To improve the crystal quality of the n-GaN thin films, the samples were subjected to thermal annealing at $\mathrm{RT}, 200{ }^{\circ} \mathrm{C}$, and $400{ }^{\circ} \mathrm{C}$ under $\mathrm{N}_{2}$ ambient for $5 \mathrm{~min}$; these samples are denoted as A, B, and C, respectively. Moreover, the undoped GaN thin film, which was denoted as sample D, was also sputtering-deposited on a glass substrate for the comparison of material quality in this study.

\subsection{Hall Measurement}

The electrical properties of the GaN samples, were investigated by using a Hall measurement with a van der Pauw configuration and using indium as the contact. The surface morphologies of the thin films were observed by atomic force microscopy (AFM).

\subsection{Photoluminescence Measurements}

The Photoluminescence (PL) measurements were performed by the employment of a He-Cd laser at a wavelength of $325 \mathrm{~nm}$ with a excitation power of $30 \mathrm{mw}$ at RT

\subsection{X-ray Diffraction (XRD) Measurement}

The X-ray diffraction (XRD) measurement was employed to study the crystallite structural properties of the $\mathrm{GaN}$ films by using a $\mathrm{Cu}-\mathrm{K} \alpha(\lambda=1.54052 \AA)$ irradiation source in a grazing incidence XRD configuration with the $2 \theta$ scan angles from $25^{\circ}$ to $70^{\circ}$. 


\subsection{Optical Transmittance Measurement}

An ultraviolet-visible spectrophotometer was employed to study the optical transmittance of the GaN samples with reference to a glass substrate in the wavelength ranges from 300 to $800 \mathrm{~nm}$.

\subsection{X-ray Photoelectron Spectroscopy Measurement}

The chemical composition of the GaN samples was analyzed at a pressure of $1.0 \times 10^{-8} \mathrm{~Pa}$ by X-ray photoelectron spectroscopy (XPS) with a Microfocus monochromatic Al K $\alpha$ radiation (1486.6 eV) source operated at $108 \mathrm{~W}$.

\section{Results and Discussion}

Figure 1 shows the X-ray diffraction (XRD) measurement results for Si-Ti-codoped GaN films of samples A, B, and C and the undoped GaN film of sample D. The XRD spectra show peaks at $2 \theta$ angles of $32.5^{\circ}$ and $34.5^{\circ}$, corresponding to the (100) and (002) crystal orientations, respectively, of the GaN films. Moreover, the dominant peak at the (002) peak of GaN thin films indicated that the sputtering-deposited GaN thin films preferentially grow with a c-axis orientation and that the crystal quality of the GaN thin films was significantly improved by thermal annealing process. Figure 1 shows that samples A, B, and C demonstrated that $2 \theta$ for the (002) crystal orientations was approximately $34.5^{\circ}$, similar with that of the undoped GaN film in sample D, indicating a slight distortion of the crystal lattice of GaN thin films after Si-Ti codoping [18,20]. Moreover, the XRD intensity of the (002) crystal orientation increased as the full width at half maximum (FWHM) decreased from $0.536^{\circ}$ to $0.418^{\circ}$, with an increase in the annealing temperature from RT to $400{ }^{\circ} \mathrm{C}$, indicating that the crystal quality improved with thermal annealing. Meanwhile, all the Si-Ti-codoped GaN films exhibited narrower FWHM values than that of the undoped GaN film in sample D, revealing the improved crystal quality by the Si-Ti-codoping technique and the thermal annealing process in this study.

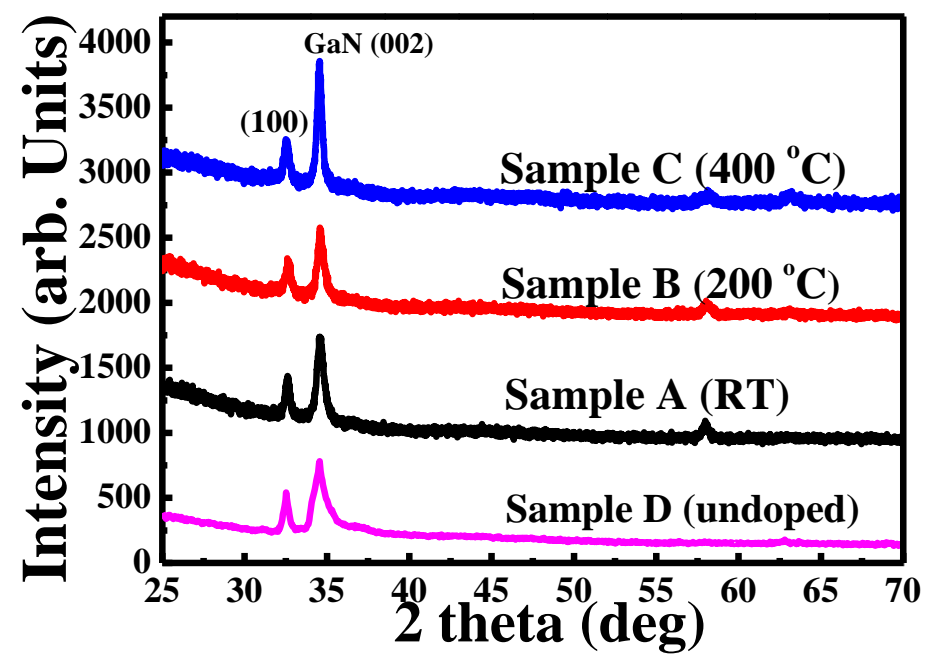

Figure 1. X-ray diffraction (XRD) pattern of silicon-titanium (Si-Ti)-codoped n-type gallium nitride $(\mathrm{GaN})$ film on a glass substrate processed at different annealing temperatures (room temperature (RT), $200^{\circ} \mathrm{C}$, and $400^{\circ} \mathrm{C}$ ). The undoped GaN film in sample D was also shown for reference.

The grain sizes of the GaN films can be determined from the FWHMs of the XRD spectra using the Scherrer formula [23]:

$$
\mathrm{D}=\frac{0.9 \lambda}{\beta \cos \theta}
$$

where $\mathrm{D}$ is the grain size, $\lambda$ the X-ray wavelength of $0.154 \mathrm{~nm}, \beta$ the XRD (002) FWHM, and $\theta$ the Bragg angle. In Figure 2, the calculated grain sizes and the corresponding XRD FWHM values of samples A, B, and C are displayed. The increased GaN crystallite size from $15.51 \mathrm{~nm}$ (sample A) 
to $19.89 \mathrm{~nm}$ (sample C) can be clearly observed, and this agrees with the AFM measurement results. At high annealing temperatures, the GaN surface adatoms possess increased thermal energy for enhanced surface diffusion, leading to the formation of more compact grains [24]. The enlarged crystallite size with increased annealing temperature as calculated from the XRD results for the GaN samples should lead to a reduction in grain-boundary scattering in the GaN thin films and, in turn, to increased carrier mobility [25].

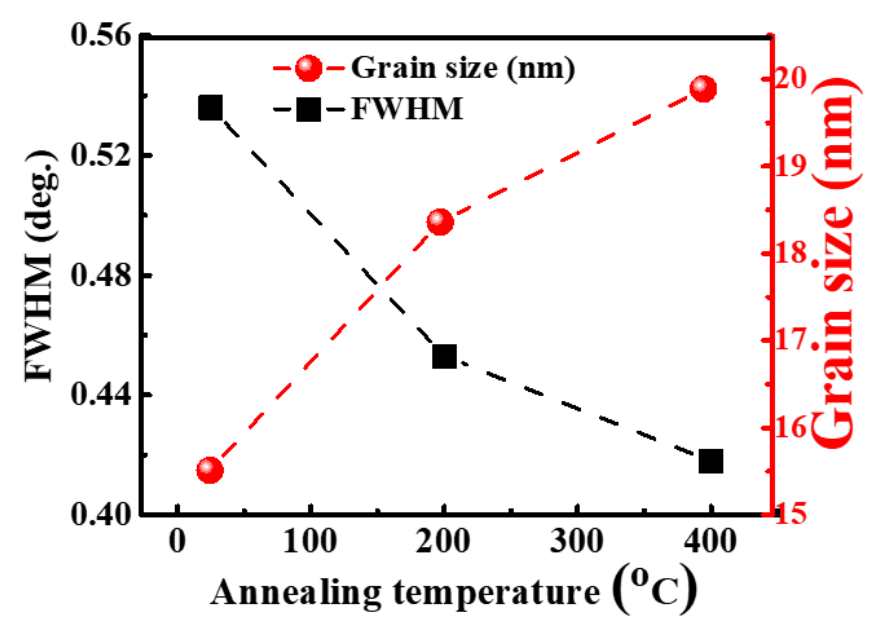

Figure 2. Plots of full width at half maximum (FWHM) values, and calculated grain size of (002) XRD of Si-Ti-codoped n-type GaN thin films at annealing temperatures of RT-400 ${ }^{\circ} \mathrm{C}$.

The carriers are easily captured by the crystal defects in the GaN films forming the negative space charge. This causes electron scattering which subsequently results in a reduction in carrier mobility and carrier concentration. Therefore, the employment of an appropriate buffer layer between the GaN film and the glass substrate is extremely important. In this study, the $\mathrm{ZnO}$ buffer layer was adopted because high crystal quality of the $\mathrm{ZnO}$ layer was easily grown on the glass substrate with the sputtering-deposition method; meanwhile, there existed a low lattice mismatch of $2.2 \%$ between the $\mathrm{ZnO}$ and $\mathrm{GaN}$ layer, which could lead to the decrease in crystal defects in the GaN films. Therefore, the adoption of $\mathrm{ZnO}$ buffer layer could improve the crystal quality of the GaN films, and thus the enhanced carrier mobility in the Hall measurement.

The electrical properties of the n-GaN thin-film samples A, B, C and un-doped GaN thin film (sample D) were determined by Hall measurements, and the results are shown in Table 1 and Figure 3. For the undoped GaN thin film of sample D, the electrical properties were observed with carrier concentration of $2.84 \times 10^{16} \mathrm{~cm}^{-3}$, carrier mobility of $0.81 \mathrm{~cm}^{2} / \mathrm{V}$-s and thin-film resistivity of $648.83 \mathrm{ohm}-\mathrm{cm}$. Compared with the sample D, the Si-Ti-codoped n-type GaN thin films in samples A show improved carrier concentration and carrier mobility. The low carrier mobility in sample D was ascribed to the scattering effects caused by considerable crystal defects and vacancies indicated by a larger FWHM value in the XRD results. In regard to incorporating the Si-Ti doping species in sputtering-deposited GaN films, the increased electron concentration was able to fill in the electron traps as well as the nonradiative recombination centers and, thus, it was screened from the defect scattering effects and contributed to the increased carrier mobility. According to the results of the Hall measurements, the electron concentration, thin-film resistivity, and carrier Hall mobility of sample A were $5.57 \times 10^{17} \mathrm{~cm}^{-3}, 11.06 \Omega-\mathrm{cm}$, and $1.02 \mathrm{~cm}^{2} / \mathrm{V}-\mathrm{s}$, respectively. The electrical properties of sample A indicate that the $\mathrm{n}-\mathrm{GaN}$ film produced by our Si-Ti-co-doped sputtering technique exhibited improved carrier concentration and thin-film resistivity in comparison to the previously reported un-doped GaN thin films. The reduced thin-film resistivity was caused by the shallow ionization energy of $30 \mathrm{meV}$ in the case of $\mathrm{Si}$ doping and by the shrinkage of the GaN energy band gap in the case of Ti doping [14,21]. In addition, after increasing the annealing temperature from RT to $400^{\circ} \mathrm{C}$ 
(i.e., in the case of sample C), the electron concentration, thin-film resistivity, and carrier Hall mobility showed pronounced improvements of $6.65 \times 10^{19} \mathrm{~cm}^{-3}, 0.26 \Omega-\mathrm{cm}$, and $14.25 \mathrm{~cm}^{2} / \mathrm{V}-\mathrm{s}$, respectively. The Hall measurement results show that sample A, which was not subjected to the thermal annealing process, had a low carrier concentration; this was because the substitution of $\mathrm{Ga}$ atoms by $\mathrm{Si}$ and $\mathrm{Ti}$ atoms was less at the lower thermal energies. Moreover, the insufficient activation of dopants in the GaN thin film that was not subjected to thermal annealing could also result from the low degree of crystallization, thus causing low carrier concentration in sample A. However, the electrical properties of sample $C$ show a significant improvement as a result of the thermal annealing process at the increased annealing temperature of $400{ }^{\circ} \mathrm{C}$. The enhancement of the electron concentration from $5.57 \times 10^{17}$ to $6.65 \times 10^{19} \mathrm{~cm}^{-3}$ in sample $\mathrm{C}$ can be attributed to the increased thermal activation of impurity dopants of group-IV elements $\left(\mathrm{Si}^{4+}\right.$ and $\left.\mathrm{Ti}^{4+}\right)$ at this high annealing temperature, which allowed them to substitute for $\mathrm{Ga}^{3+}$, thereby providing additional electrons in the n-GaN thin films [26].

Table 1. Hall measurement value of Si-Ti-codoped GaN processed at different annealing temperatures (RT, $200{ }^{\circ} \mathrm{C}$, and $400^{\circ} \mathrm{C}$ ).

\begin{tabular}{cccc}
\hline Sample & Resistivity $(\boldsymbol{\Omega}$-cm) & Carrier Concentration $\left(\mathbf{c m}^{-3}\right)$ & Mobility $\left(\mathbf{c m}^{2} / \mathbf{V}\right.$-s) \\
\hline Sample A $(\mathrm{RT})$ & 11.06 & $5.57 \times 10^{17}$ & 1.02 \\
Sample B $\left(200^{\circ} \mathrm{C}\right)$ & 3.32 & $8.06 \times 10^{18}$ & 8.52 \\
Sample C $\left(400^{\circ} \mathrm{C}\right)$ & 0.26 & $6.65 \times 10^{19}$ & 14.25 \\
Sample D (undoped) & 648.83 & $2.84 \times 10^{16}$ & 0.81 \\
\hline
\end{tabular}

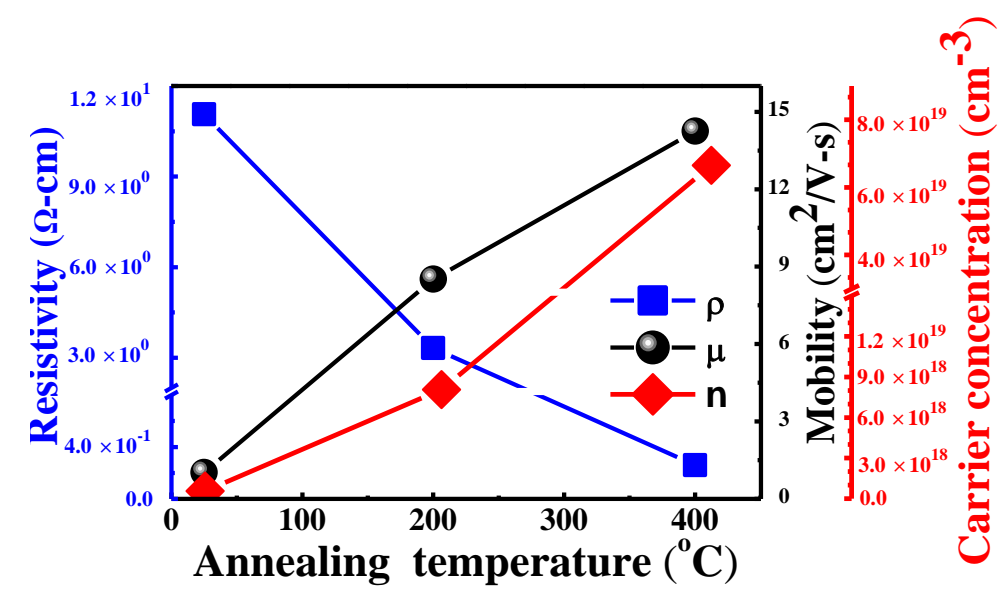

Figure 3. Hall measurements of Si-Ti-codoped n-type GaN films processed at different annealing temperatures (RT, $200{ }^{\circ} \mathrm{C}$, and $400{ }^{\circ} \mathrm{C}$ ): $\mu$ is the mobility, $\mathrm{n}$ is the carrier concentration, and $\rho$ is the resistivity.

The low carrier mobility of sample A could furthermore result from the prominent boundary scattering in GaN samples with insufficient thermal energies that would result in high thin-film resistivity. However, the carrier mobility of the GaN thin film can be improved by increasing the annealing temperature. In this work, the annealing process was adopted to improve the carrier mobility from $1.02 \mathrm{~cm}^{2} / \mathrm{V}$-s (sample A) to $14.25 \mathrm{~cm}^{2} / \mathrm{V}$-s (sample C) because of the reduction in the carrier scattering effect associated with the reduced crystal defects of the GaN film with an elevated annealing temperature of $400{ }^{\circ} \mathrm{C}$. In addition, the increased carrier concentration was also due to the reduced activation energy and reduced nonradiative recombination centers for an improved GaN crystal quality. Hence, the increase in carrier mobility and carrier concentration combined with the increased annealing temperature indicated the improvement of the crystalline quality [27]. In addition, GaN thin-film crystallites were able to be more compact and denser in sample $C$ due to the increased thermal energies at elevated annealing temperatures. An enlarged crystallite size of the $\mathrm{GaN}$ film in sample $\mathrm{C}$ with its high annealing temperature could also contribute to the reduction in grain-boundary scattering, 
resulting in the increased carrier mobility in sample C; this, in turn, would result in the electrical resistivity decreasing from 11.06 to $0.26 \Omega-\mathrm{cm}$.

The surface morphology of the Si-Ti-codoped and undoped GaN films for samples A, B, C, and D was analyzed by atomic force microscopy (AFM) as shown in Figure 4a-d, respectively. The grain sizes of the GaN films were measured as $13.89,17.61,18.13 \mathrm{~nm}$, and 11.75 for the GaN thin films in samples A, B, C, and D, respectively. The small grain size of samples A and D (without any annealing) was due to insufficient thermal energy and subsequent low surface diffusion of adatoms for crystal growth; this results in small crystallite formation. The increase in grain size of the GaN thin films to $18.13 \mathrm{~nm}$ as the annealing temperature increased from RT to $400{ }^{\circ} \mathrm{C}$ was due to crystallite interaction and aggregation at high annealing temperatures. Moreover, the AFM measurement demonstrated that the film roughness decreased from 3.57 to $2.80 \mathrm{~nm}$ as the annealing temperature increased from RT to $400{ }^{\circ} \mathrm{C}$, while the surface roughness was $4.12 \mathrm{~nm}$ for sample $\mathrm{D}$. The tendency toward decreased surface roughness is attributable to the increased thermal energy and enhanced diffusion of surface atoms, which could lead to enhanced crystallite growth, resulting in enlarged grain size and dense, flat thin-film growth. Therefore, according to the AFM data, the GaN thin film in sample C demonstrated the largest grain size $(18.13 \mathrm{~nm})$ and the smallest roughness $(2.80 \mathrm{~nm})$.
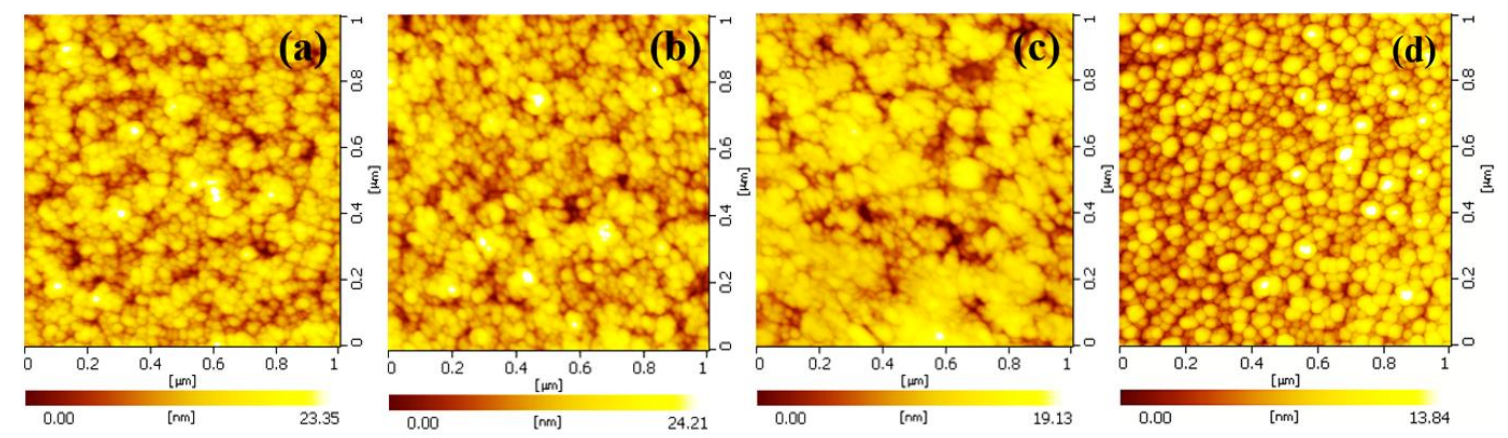

Figure 4. Atomic force microscopy (AFM) measurement results of samples (a) A, (b) B, (c) C, and (d) D.

In Figure 5, the results of RT photoluminescence (PL) measurements of samples A, B, C and D are presented. The optical properties, energy bandgap, and carrier energy transition behavior in n-type GaN thin films were analyzed in detail. As shown in Figure 5, GaN films exhibit two dominant emission peaks at around 365 and $413 \mathrm{~nm}$. The peak signal at $365 \mathrm{~nm}$ is related to the near-band-edge (NBE) emission for the carrier transition from the valence band to the lowest allowed energy states in the conduction band of the GaN thin film with a hexagonal crystal structure [28]. The typical blue luminescence (BL) band in GaN thin films was observed at the peak position in the range from 3.0 to $2.7 \mathrm{eV}$, and the dominant BL peak in this study was at $3.0 \mathrm{eV}(413 \mathrm{~nm})$. The PL emission peak at $413 \mathrm{~nm}$ can be ascribed to the energy transition from the conduction band or a shallow donor level to a deep acceptor level. Density functional theory calculations demonstrated the carrier transition from the $\mathrm{C}_{\mathrm{Ga}}$ donor level to the $\mathrm{C}_{\mathrm{N}}$ acceptor level because the carbon impurities in the GaN films can account for the BL band at the PL peak signal of approximately $413 \mathrm{~nm}(3.0 \mathrm{eV})[29,30]$. The considerable Carbon (C) impurity may be attributable to the fact that the sputtering system employed here belongs to a public instrument; therefore, a variety of material systems are fabricated in the same chamber (e.g., Silicon Carbide (SiC) and other C-containing materials). Therefore, in this study, the GaN films demonstrated $\mathrm{C}$ contamination, resulting in a substantial amount of unexpected impurities in them. As shown in Figure 5, the intensity of the NBE emission peak signal was enhanced by performing a thermal annealing process that elevated the temperature from RT to $400{ }^{\circ} \mathrm{C}$; this indicates that enhanced crystallization increased the GaN grain size, thereby improving the GaN crystal quality. In addition, Figure 5 demonstrates that the intensity of the peak signal at $413 \mathrm{~nm}(3.0 \mathrm{eV})$ depended on the annealing temperature. This could be related to enhanced $\mathrm{Ga}$ atom substitution by $\mathrm{C}$ impurities at 
high annealing temperatures, leading to an increased number of carrier transitions from the $\mathrm{C}_{\mathrm{Ga}}$ donor level to the $\mathrm{C}_{\mathrm{N}}$ acceptor level as the annealing temperature increased.

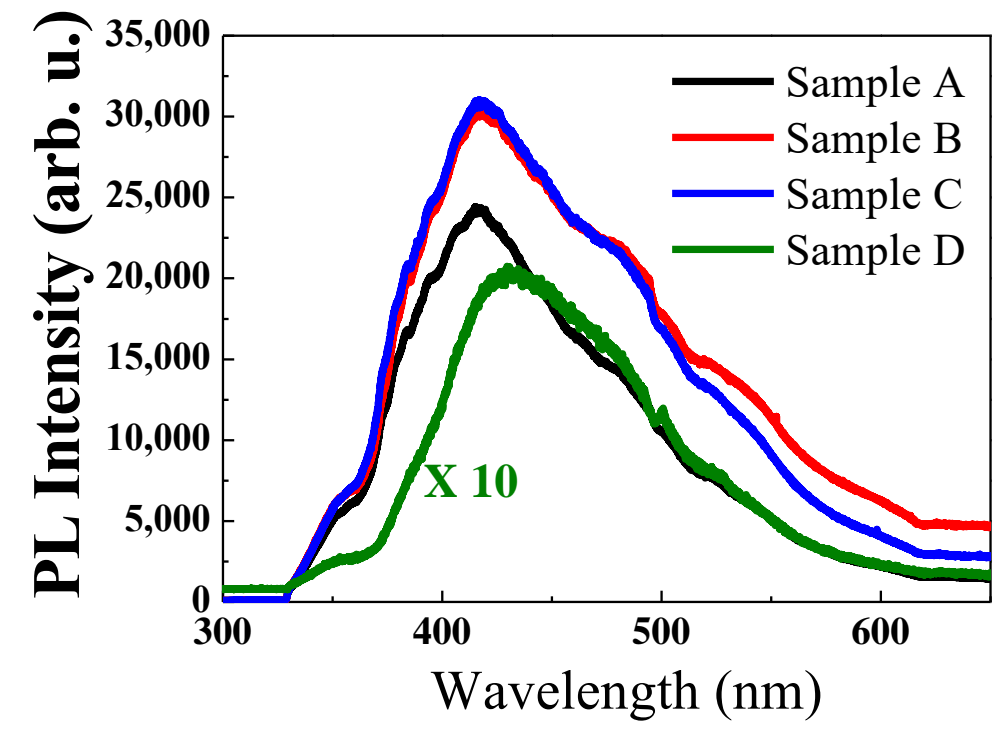

Figure 5. Photoluminescence (PL) spectra of the Si-Ti-codoped GaN films in samples A, B, C and the undoped GaN film in Sample D.

Additionally, the PL intensity of the NBE emission for the Si-Ti-codoped GaN films were significantly increased compared with that of the undoped GaN film in sample D, indicating the improved crystal quality by the Si-Ti-codoping technique in samples A, B and C. The improved crystal quality by the PL measurement was also agreed well with the study results of the XRD measurement in Figure 1.

Figure 6 shows the transmittance of GaN thin-film samples A, B, and C in the range of 300 to $800 \mathrm{~nm}$. The average transmittances of the Si-Ti-codoped GaN film were 81.94, 83.53, and 85.51\% at annealing temperatures of RT, $200{ }^{\circ} \mathrm{C}$, and $400{ }^{\circ} \mathrm{C}$, respectively. As shown in Figure $6 \mathrm{a}$, the absorption edge for all GaN samples occurs at approximately $365 \mathrm{~nm}$. Since the GaN thin films deposited by the sputtering system exhibited the polycrystalline structure, the grain-boundary scattering could be the dominant effects responsible for the transmittance. The grain size of the GaN film was found to be increased when increasing the annealing temperatures from RT to $400{ }^{\circ} \mathrm{C}$, as evident in the AFM and XRD studies. Hence, the reduction in the optical scattering by the reduced grain boundaries could contribute to the increase in the optical transmittance in the dense GaN films with enlarged grain size [31]. Additionally, through the AFM measurement, it can be observed that sample C exhibit the reduced surface roughness compared with the other GaN samples, which could also lead to the increased transmittance due to the reduced surface scattering [32]. To investigate the relationship between the optical and the electronic properties of the GaN thin films, a Tauc plot was calculated using Equation (2) and the data from the transmittance measurement:

$$
(\alpha \mathrm{h} v)^{1 / \mathrm{n}}=\mathrm{A}\left(\mathrm{h} v-\mathrm{E}_{\mathrm{g}}\right)
$$

where $\alpha$ is the absorption coefficient, h Planck's constant, $v$ the photon frequency, A a proportionality constant, and $E_{g}$ the energy bandgap of the material. Furthermore, $n=1 / 2$ was adopted here because of the direct transitions of GaN films. As displayed in Figure 6b, the optical bandgap values of the GaN films exhibited an increasing trend from 3.33 to $3.41 \mathrm{eV}$ for samples $\mathrm{A}$ to $\mathrm{C}$ as the annealing temperatures increased from RT to $400{ }^{\circ} \mathrm{C}$. In addition, the transmittance edge shifted to higher bandgap from 3.33 to $3.41 \mathrm{~nm}$ for samples A to C; this might be due to the Burstein-Moss effect. The Burstein-Moss effect resulted from the high doping concentration which will cause the blue shift of 
the optical absorption edge. As the distribution of the degenerated semiconductor's electron is full at the near-band edge of the conduction band, it causes the higher transition energy from the valence band. Hence, the tendency of the enlarge bandgap or the blue shift of the optical absorption edge was named the Burstein-Moss shift $[33,34]$. According to Lee's report, they found that the photoluminescence peak signal position of the highly compensated Si-doped GaN film decreased as the electron concentration increased, which was due to the forbidden band-edge fluctuation resulting from residual impurities [35]. Moreover, the band gap renormalization was similar to the band gap narrowing effect. These can be explained by the decrease in the bandgap as the doping concentration rises due to its self-energy effect [36]. Notably, the Fermi level inside the conduction band moves upward, indicating an increased carrier concentration of the GaN films with an annealing temperatures ranging from RT to $400{ }^{\circ} \mathrm{C}$ [37]. This trend in the Tauc plot results agrees with the effects of an increased electron concentration in the Hall measurement.
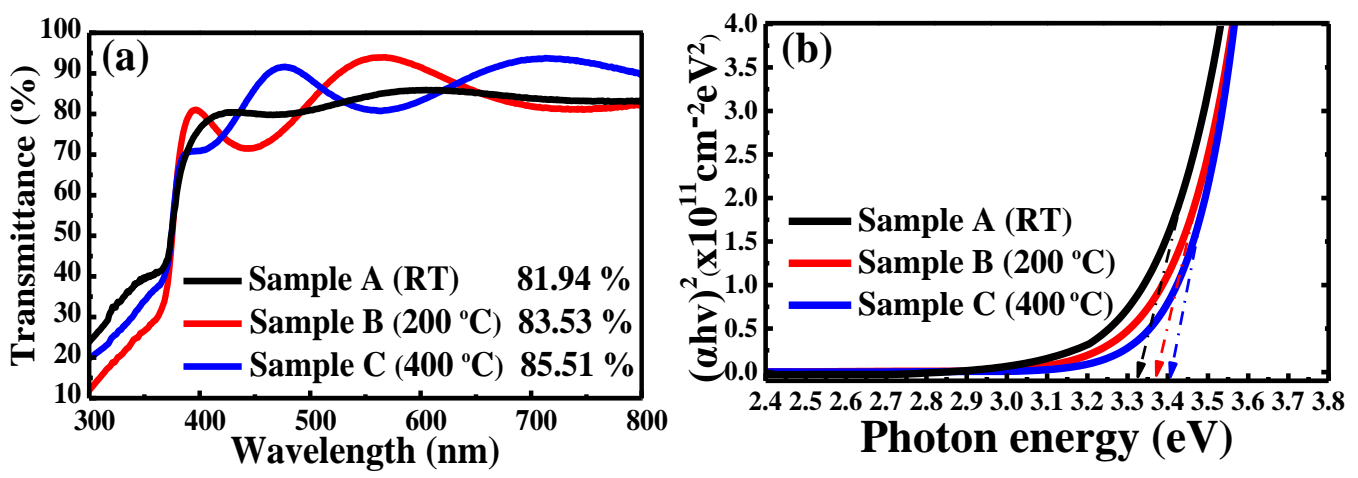

Figure 6. (a) Transmittance and (b) optical bandgap of Si-Ti-codoped n-type GaN film at different annealing temperatures (RT, $200^{\circ} \mathrm{C}$, and $400^{\circ} \mathrm{C}$ ).

The chemical composition of the Si-Ti-codoped GaN film annealed at $400{ }^{\circ} \mathrm{C}$ was analyzed by X-ray photoelectron spectroscopy (XPS), as shown in Figure 7. The binding energies of Gallium on the $3 d$ orbital (Ga-3d), Silicon on the 2p orbital (Si-2p), Nitrogen on the 1s orbital (N-1s), Titanium on the $2 \mathrm{p}$ orbital (Ti-2p), and Gallium on the $2 \mathrm{p}_{2 / 3}$ orbital (Ga-2 $\left.\mathrm{p}_{3 / 2}\right)$ were 19.7, 102.6, 397.3, 455.6, and $1119.5 \mathrm{eV}$, respectively. The $\mathrm{C}-1 \mathrm{~s}$ spectrum was calibrated at $284.6 \mathrm{eV}$ as a reference element for all signals. The high-resolution Ga-3d XPS spectra with fitting curves showed that the Ga-3d spectrum consisted of the Ga-Ga bond, the Ga-N bond, and the Ga-O bond; the binding energies of these were 18.2, 19.7, and $20.5 \mathrm{eV}$, respectively, as shown in Figure 8a. These binding energy values are in agreement with other reported results [38]. The Si-2p spectrum with fitting curves shown in Figure $8 b$ was composed of the Si-Si bond, the $\mathrm{Si}-\mathrm{N}$ bond [39], and the $\mathrm{Ga}-\mathrm{N}$ bond [40] with corresponding binding energies of 99.1, 102.6, and $106.1 \mathrm{eV}$. The appearance of the Ga-N bond in the Si-2p spectrum can be ascribed to the similarity in the binding energy values between Si-2p and Ga- $3 p_{1 / 2}$. The N-1s spectrum shown in Figure 8c is made up of two peaks corresponding to the binding energies of 392.7 and $397.3 \mathrm{eV}$, respectively. The binding energy at $392.7 \mathrm{eV}$ can be attributed to the Ga LMM Auger transitions that revealed the presence of metallic-like Ga [41]. The peak at $397.3 \mathrm{eV}$ appearing in the N-1s spectrum corresponds to the bonding between gallium and nitrogen atoms, thus forming the Ga-N bond. In addition, it can be seen in Figure $8 \mathrm{~d}$ that Ga-2 $\mathrm{p}_{3 / 2}$ has a dominant peak at $1118.7 \mathrm{eV}$ and a smaller peak at $1119.5 \mathrm{eV}$. 


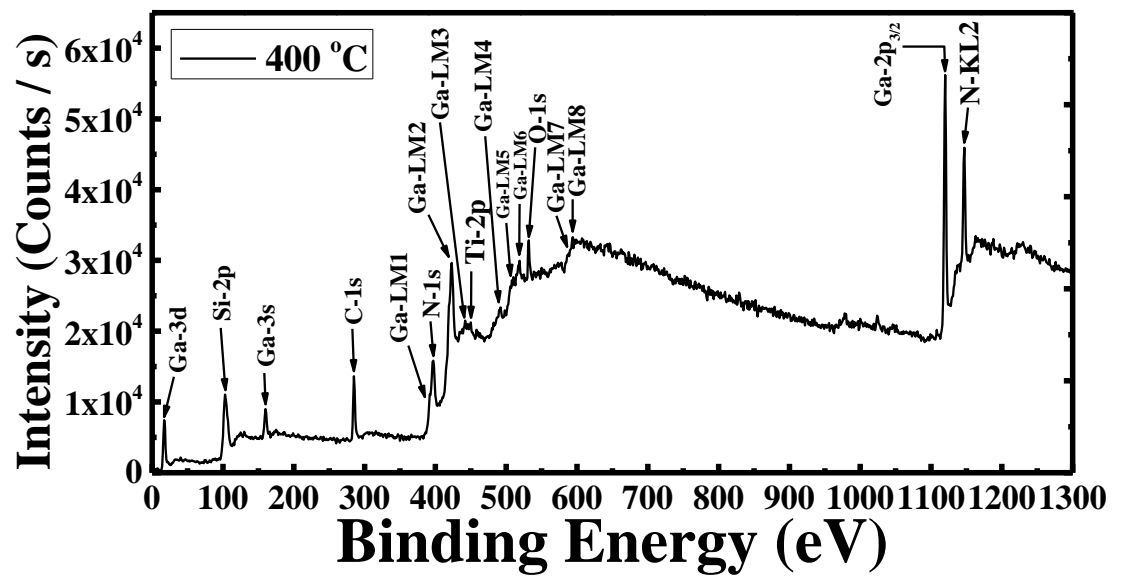

Figure 7. X-ray photoelectron spectra survey scan of Si-Ti-codoped n-type GaN film processed at the annealing temperature of sample $C$.
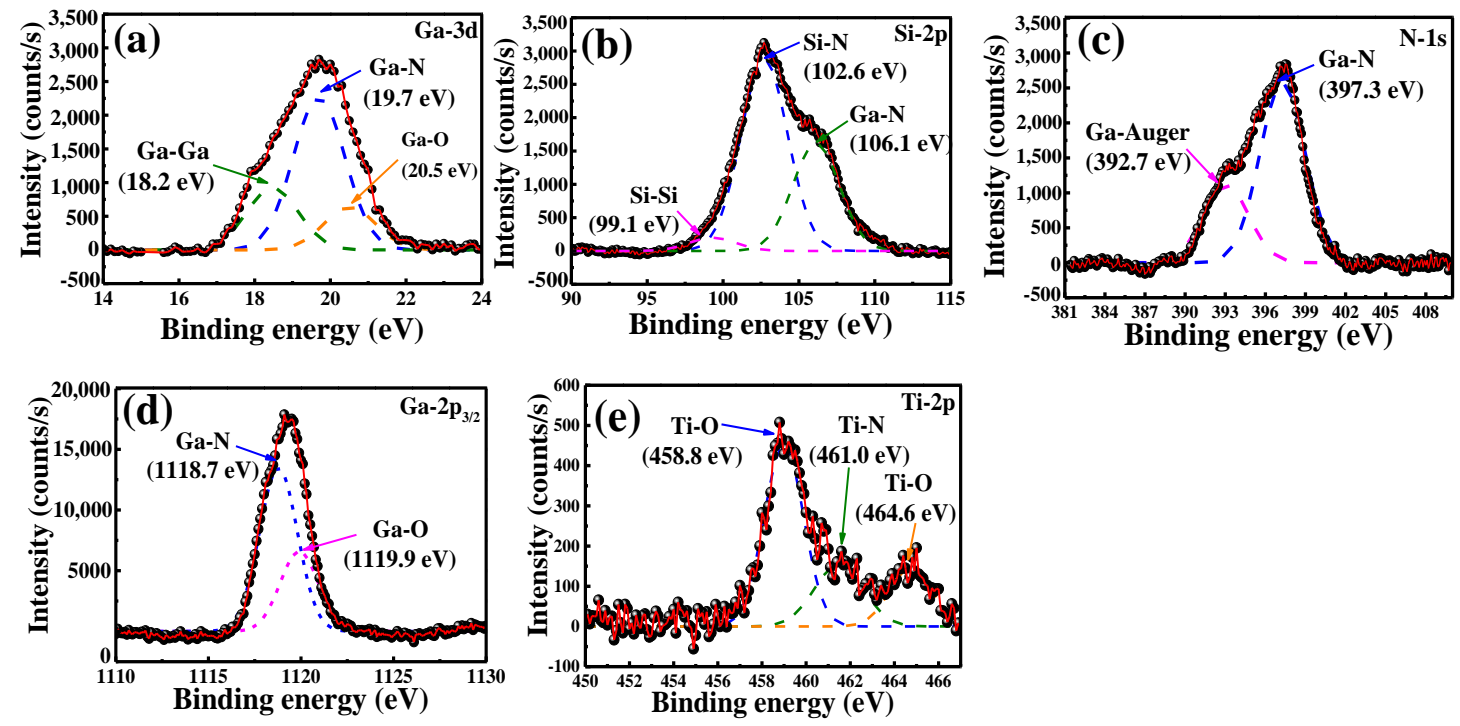

Figure 8. (a) Ga-3d X-ray photoelectron spectroscopy (XPS) spectra; (b) Si-2p XPS spectra; (c) N-1s spectra; (d) Ga-2 $p_{3 / 2}$ XPS spectra; (e) Ti-2p XPS spectra with fitting curve results obtained from the Si-Ti-codoped n-type GaN film processed at the annealing temperature of sample $\mathrm{C}$.

The binding energy at $1118.7 \mathrm{eV}$ could be ascribed to the bonding between the $\mathrm{Ga}-\mathrm{N}$ components while the binding energy at $1119.5 \mathrm{eV}$ corresponds to the $\mathrm{Ga}-\mathrm{O}$ bond [42,43]. According to Dasari's report, the binding energy standard of the $\mathrm{Ga}-2 \mathrm{p}_{3 / 2}$ was $1117 \mathrm{eV}$, which is lower than our result, and it can be ascribed to the formation of a nitride compound. This result of Ga-2 $\mathrm{p}_{2 / 3}$ agrees with the result of Ga-3d, which appears to have a greater amount of the Ga-N bond than the Ga-O bond [44]. As shown in Figure 8e, the XPS peak belonging to Ti-2p was analyzed based on the XPS spectral survey scan, and the binding energy of both Ti-O, and Ti-N was $458.8,464.6 \mathrm{eV}$ and $461.0 \mathrm{eV}$, respectively $[45,46]$. The high XPS intensity in Ti-O bonding might be due to the Ti bonding with the oxide residue during the sputtering-deposition of $\mathrm{ZnO}$ buffer layer. The high XPS intensity of the Ga-O bond in the GaN thin film might be due to the unintentional oxygen adsorption of the $\mathrm{GaN}$ thin-film surface and the residual oxide compound in the growth chamber after the sputtering deposition of the $\mathrm{ZnO}$ buffer layer. The XPS measurement results indicate that all atoms doped into the GaN film are present and that both $\mathrm{Si}$ and Ti bond with $\mathrm{N}$ atoms. 


\section{Conclusions}

In this study, we developed a Si-Ti-codoped n-type GaN thin film with a $\mathrm{ZnO}$ buffer layer on an amorphous glass substrate by using a cosputtering system followed by thermal annealing for optimizing GaN crystal quality. The experimental results indicate that the n-type GaN thin film subjected to thermal annealing at $400{ }^{\circ} \mathrm{C}$ exhibited the greatest crystal growth among the $\mathrm{GaN}$ samples. Hall measurement results indicated that $\mathrm{GaN}$ sample $\mathrm{C}$ has the lowest thin-film resistivity at $2.6 \times 10^{-1} \Omega-\mathrm{cm}$, highest mobility at $14.25 \mathrm{~cm}^{2} / \mathrm{V}-\mathrm{s}$, and highest concentration at $6.65 \times 10^{19} \mathrm{~cm}^{-3}$ among all GaN samples. The PL measurement results indicated a typical NBE emission wavelength was in accordance with the hexagonal GaN crystal structure at $365 \mathrm{~nm}$ with a deep defect level emission peak at $413 \mathrm{~nm}$. The deep defect level emission peak at $413 \mathrm{~nm}$ can be ascribed to the carrier transition from the CGa donor level to the $\mathrm{CN}$ acceptor level due to the carbon impurity in the GaN films. PL measurement results indicated that a high-quality n-type GaN thin film can be developed with a decrease in the deep defect level. The XRD measurement showed that $2 \theta$ for (002) crystal orientations was approximately $34.5^{\circ}$ for all the GaN samples of undoped GaN thin film and samples A, B, and C, which indicating a slight distortion of the crystal lattice of $\mathrm{GaN}$ thin films after the Si-Ti-codoping sputtering process. Furthermore, calculations using the Scherrer formula indicated that the calculated grain size values were similar to the AFM measurement results.

Due to the enlarged crystallite size calculated from the XRD results of GaN samples with increasing annealing temperature, the reduction in the grain-boundary scattering of the GaN thin films led to an increased carrier mobility and enhanced average transmittance. Moreover, the Burstein-Moss effect was observed in the Tauc plot results, indicating that the Fermi level inside the conduction band moves upward, and thus improves the n-type properties of the GaN thin film. XPS measurement results indicated that all atoms doped into the GaN film are present and that both $\mathrm{Si}$ and $\mathrm{Ti}$ atoms bond with $\mathrm{N}$ atoms. In conclusion, the study results indicated that high-quality n-type GaN films could be fabricated by a Si-Ti-codoping sputtering technique with a $\mathrm{ZnO}$ buffer layer on an amorphous glass substrates followed by thermal annealing. This process is a potential candidate for large-scale and low-cost fabrication of GaN-related optoelectronic devices.

Author Contributions: Conceptualization, W.-S.L., Y. -L.C., and C.-Y.T.; methodology, Y.-L.C., C.-Y.T., and C.-T.T.; validation, Y.-L.C., C.-Y.T., and C.-T.T.; formal analysis, Y.-L.C., C.-Y.T., and C.-T.T.; investigation, W.-S.L.; resources, W.-S.L. and H.-C.K.; data curation, Y.-L.C., C.-Y.T., and C.-T.T.; writing-original draft preparation, Y.-L.C., C.-Y.T., and C.-T.T.; writing - review and Editing, W.-S.L. and H.-C.K.; visualization, W.-S.L.; supervision, W.-S.L.; project administration, W.-S.L.; funding acquisition, W.-S.L and H.-C.K. All authors have read and agreed to the published version of the manuscript.

Funding: This research was funded by Ministry of Science and Technology, Taiwan, grant number MOST106-2221-E-155-041-MY3, MOST 109-2221-E-155-050 and Chang Gung Memorial Hospital, Chiayi, Taiwan, grant number BMRPD42.

Acknowledgments: The authors are grateful to Ministry of Science and Technology, Taiwan, for financial support under contracts MOST106-2221-E-155-041-MY3 and MOST 109-2221-E-155-050. The funding grant of BMRPD42 for this study was also provided in part by research grants from the Chang Gung Memorial Hospital, Chiayi, Taiwan. The provision of research equipment by the Optical Sciences Center at National Central University is greatly appreciated.

Conflicts of Interest: The authors declare no conflict of interest.

\section{References}

1. Vurgaftman, I.; Meyer, J.R.; Ram-Mohan, L.R. Band parameters for III-V compound semiconductors and their alloys. J. Appl. Phys. 2001, 89, 5815-5875. [CrossRef]

2. Jain, S.C.; Willander, M.; Narayan, J.; van Overstraeten, R. III-nitrides: Growth, characterization, and Properties. J. Appl. Phys. 2000, 87, 965-1006. [CrossRef]

3. Somanathan, N.; Sambathkumar, B.; Ananthakrishnan, S.J. Studies on polythiophenes containing fused polyaromatic rings as side chains. Eur. Polym. J. 2014, 52, 23-31. [CrossRef] 
4. Tu, W.; Liu, T.; Zhang, Z.; Wu, G.; Chen, H.; Wang, M. Ultra-wide bandgap organic acceptor material and its application in organic UV photodetector. Synth. Met. 2016, 219, 20-25. [CrossRef]

5. Bouarissa, N. Pressure dependence of optoelectronic properties of GaN in the zinc-blende structure. Mater. Chem. Phys. 2002, 73, 51-56. [CrossRef]

6. Ma, R.; Teo, K.H.; Shinjo, S.; Yamanaka, K.; Asbeck, P. A GaN PA for 4G LTE-Advanced and 5G. IEEE Microw. Mag. 2017, 18, 77-85. [CrossRef]

7. Wickenden, A.E.; Koleske1, D.D.; Henry, R.L.; Twigg, M.E.; Fatemi, M. Resistivity control in unintentionally doped GaN films grown by MOCVD. J. Cryst. Growth 2004, 260, 54-62. [CrossRef]

8. Andre, Y.; Trassoudaine, A.; Tourret, J.; Cadoret, R.; Gil, E.; Castelluci, D.; Aoude, O.; Disseix, P. Low dislocation density high-quality thick hydride vapour phase epitaxy (HVPE) GaN layers. J. Cryst. Growth 2007, 306, 86-93. [CrossRef]

9. Kocan, M.; Rizzi, A.; Luth, H.; Keller, S.; Mishra, U.K. Surface Potential at as-Grown GaN(0001) MBE Layers. Phys. Stat. Sol. (B) 2002, 234, 773-777. [CrossRef]

10. Shon, J.W.; Ohta, J.; Ueno, K.; Kobayashi, A.; Fujioka, H. Structural properties of GaN films grown on multilayer graphene films by pulsed sputtering. Appl. Phys. Express 2014, 7, 085502. [CrossRef]

11. Zou, C.W.; Wang, H.J.; Yin, M.L.; Li, M.; Liu, C.S.; Guo, L.P.; Fu, D.J.; Kang, T.W. Preparation of GaN films on glass substrates by middle frequency Magnetron sputtering. J. Cryst. Growth 2009, 311, 223-227. [CrossRef]

12. Zou, C.W.; Yin, M.L.; Li, M.; Liu, C.S.; Guo, L.P.; Fu, D.J. GaN films deposited on glass substrate by middle-frequency magnetron sputtering. Thin Solid Film. 2008, 517, 670-673. [CrossRef]

13. Jonsson, L.B.; Nyberg, T.; Katardjiev, I.; Berg, S. Frequency response in pulsed DC reactive sputtering processes. Thin Solid Film. 2000, 365, 43-48. [CrossRef]

14. Shikanai, A.; Fukahori, H.; Kawakami, Y.; Hazu, K.; Sota, T.; Mitani, T.; Mukai, T.; Fujita, S. Optical properties of Si-, Ge- and Sn-doped GaN. Phys. Stat. Sol. (B) 2003, 235, 26-30. [CrossRef]

15. Romano, L.T.; Van de Walle, C.G.; Ager III, J.W.; Götz, W.; Kern, R.S. Effect of Si doping on strain, cracking, and microstructure in GaN thin films grown by metalorganic chemical vapor deposition. J. Appl. Phys. 2000, 87, 7745-7752. [CrossRef]

16. Shuji, N.; Takashi, M.; Senoh, M. Si- and Ge-Doped GaN Films Grown with GaN Buffer Layers. Jpn. J. Appl. Phys. 1992, 31, 2883-2888.

17. Xu, Z.; Zhang, J.; Duan, H.; Zhang, Z.; Zhu, Q.; Xu, H.; Hao, Y. Stress structural and electrical properties of Si-doped GaN film grown by MOCVD. J. Semicond. 2009, 30, 123003.

18. Cui, Z.; Ke, X.; Li, E.; Liu, T. Electronic and optical properties of titanium-doped GaN nanowires. Mater. Des. 2016, 96, 409-415. [CrossRef]

19. Mendoza-Estrada, V.; González-García, A.; López-Pérez, W.; Pinilla, C.; González-Hernández, R. Structural, electronic and magnetic properties of Ti-doped polar and nonpolar GaN surfaces. J. Cryst. Growth 2017, 467, 12-17. [CrossRef]

20. Lu, C.; Chen, H.; Lv, X.; Xie, X.; Mohammad, S.N. Temperature and doping-dependent resistivity of $\mathrm{Ti} / \mathrm{Au} / \mathrm{Pd} / \mathrm{Au}$ multilayer ohmic contact to n-GaN. J. Appl. Phys. 2002, 91, 9218-9224. [CrossRef]

21. Majid, A.; Javed, M.; Rana, U.A.; Khan, S.U.-D. TiGa-VN complexes in GaN: A new prospect of carrier mediated ferromagnetism. RSC Adv. 2015, 5, 87437-87444. [CrossRef]

22. Xiong, Z.; Shi, S.; Jiang, F. Ti in GaN: Ordering ferromagnetically from first-principles study. Chem. Phys. Lett. 2007, 443, 92-94. [CrossRef]

23. Patterson, A.L. The Scherrer Formula for I-Ray Particle Size Determination. Phys. Rev. 1939, 56, $978-982$. [CrossRef]

24. Liu, W.-S.; Hsieh, W.-T.; Chen, S.-Y.; Huang, C.-S. Improvement of CIGS solar cells with high performance transparent conducting Ti-doped GaZnO thin films. Sol. Energy 2018, 174, 83-96. [CrossRef]

25. Lv, M.; Xiu, X.; Pang, Z.; Dai, Y.; Han, S. Influence of the deposition pressure on the properties of transparent conducting zirconium-doped zinc oxide films prepared by RF magnetron sputtering. Appl. Surf. Sci. 2006, 252, 5687-5692. [CrossRef]

26. Liu, W.-S.; Wu, S.-Y.; Tseng, C.-H.; Hung, C.-Y. Quality improvement of high-performance transparent conductive Ti-doped GaZnO thin film. Thin Solid Film. 2014, 570, 568-573. [CrossRef]

27. Cole, M.W.; Ren, F.; Pearton, S.J. Post growth rapid thermal annealing of GaN: The relationship between annealing temperature, GaN crystal quality, and contact-GaN interfacial structure. Appl. Phys. Lett. 1997, 71, 3004-3006. [CrossRef] 
28. Pankove, J.I.; Schade, H. Photoemission from GaN. Appl. Phys. Lett. 1974, 25, 53-55. [CrossRef]

29. Reshchikov, M.A.; Moon, Y.T.; Morkoç, H. Origin of unstable photoluminescence in GaN: Metastable defects or surface states. Phys. Stat. Sol. (C) 2005, 2, 2716-2719. [CrossRef]

30. Liu, W.-S.; Chang, Y.-L.; Chen, H.-Y. Growth of GaN Thin Film on Amorphous Glass Substrate by Direct-Current Pulse Sputtering Deposition Technique. Coatings 2019, 9, 419. [CrossRef]

31. Kumari, V.; Kumar, V.; Mohan, D.; Purnima; Malik, B.P.; Mehra, R.M. Effect of Surface Roughness on Laser Induced Nonlinear Optical Properties of Annealed ZnO Thin Films. J. Mater. Sci. Technol. 2012, 28, 506-511. [CrossRef]

32. Jain, V.K.; Kumar, P.; Vijay, Y.K. Preparation of Nanostructure $\mathrm{ZnO}-\mathrm{SnO}_{2}$ Thin Films for Optoelectronic Properties and Post Annealing Influence. Int. Sch. Sci. Res. Innov. 2012, 6, 1192-1194.

33. Burstein, E. Anoma1ous Optical Absorption Limit in InSb. Phys. Rev. 1954, 93, 632-633. [CrossRef]

34. Moss, T.S. The Interpretation of the Properties of Indium Antimonide. Proc. Phys. Soc. Lond. B 1954, 67, 775-782. [CrossRef]

35. Lee, I.-H.; Lee, J.J.; Kung, P.; Sanchez, F.J.; Razeghi, M. Band-gap narrowing and potential fluctuation in Si-doped GaN. Appl. Phys. Lett. 1999, 74, 102-104. [CrossRef]

36. Yoshikawa, M.; Kunzer, M.; Wagner, J.; Obloh, H.; Schlotter, P.; Schmidt, R.; Herres, N.; Kaufmann, U. Band-gap renormalization and band filling in Si-doped GaN films studied by photoluminescence spectroscopy. J. Appl. Phys. 1999, 86, 4400-4402. [CrossRef]

37. Pu, N.-W.; Liu, W.-S.; Cheng, H.-M.; Hu, H.-C.; Hsieh, W.-T.; Yu, H.-W.; Liang, S.-C. Investigation of the Optoelectronic Properties of Ti-doped Indium Tin Oxide Thin Film. Materials 2015, 8, 6471-6481. [CrossRef]

38. Thakur, V.; Shivaprasad, S.M. Electronic structure of GaN nanowall network analysed by XPS. Appl. Surf. Sci. 2015, 327, 389-393. [CrossRef]

39. Kumar, M.; Roul, B.; Thirumaleshwara, N.B.; Mohana, K.R.; Kalghatgi, A.T.; Krupanidhi, S.B. Valence band offset at $\mathrm{GaN} / \beta-\mathrm{Si}_{3} \mathrm{~N}_{4}$ and $\beta-\mathrm{Si}_{3} \mathrm{~N}_{4} / \mathrm{Si}$ (111) heterojunctions formed by plasma-assisted molecular beam epitaxy. Thin Solid Film. 2012, 520, 4911-4915. [CrossRef]

40. Martin, G.; Botchkarev, A.; Rockett, A.; Morkoç, H. Valenceband discontinuities of wurtzite GaN, AlN, and InN heterojunctions measured by xray photoemission spectroscopy. Appl. Phys. Lett. 1996, 68, 2541-2543. [CrossRef]

41. Berto' ti, I. Characterization of nitride coatings by XPS. Surf. Coat. Technol. 2002, 151, 194-203. [CrossRef]

42. Xue, S.; Zhang, X.; Huang, R.; Zhuang, H.; Xue, C. Defect-pit-assisted growth of GaN nanostructures: Nanowires, nanorods and nanobelts. R. Soc. Chem. 2008, 32, 4296-4302. [CrossRef] [PubMed]

43. Chen, Z.; Wang, X.; Noda, S.; Saito, K.; Tanaka, T.; Nishio, M.; Arita, M.; Guo, Q. Effects of dopant contents on structural, morphological and optical properties of Er doped $\mathrm{Ga}_{2} \mathrm{O}_{3}$ films. Superlattices Microstruct. 2016, 90, 207-214. [CrossRef]

44. Dasari, K.; Wu, J.; Huhtinen, H.; Jadwisienczak, W.M.; Palai, R. Growth luminescence and magnetic properties of GaN:Er semiconductor thin films grown by molecular beam epitaxy. J. Phys. D Appl. Phys. 2017, 50, 175104. [CrossRef]

45. Jung, S.-H.; Kang, S.-W. Formation of $\mathrm{TiO}_{2}$ thin films using $\mathrm{NH} 3$ as Catalyst by Metalorganic Chemical Vapor Deposition. Jpn. J. Appl. Phys. 2001, 40, 3147-3152. [CrossRef]

46. Höchst, H.; Bringans, R.D.; Steiner, P.; Wolf, T. Photoemission study of the electronic structure of stoichiometric and substoichiometric TiN and ZrN. Phys. Rev. B 1982, 25, 7183-7191. [CrossRef]

(C) 2020 by the authors. Licensee MDPI, Basel, Switzerland. This article is an open access article distributed under the terms and conditions of the Creative Commons Attribution (CC BY) license (http://creativecommons.org/licenses/by/4.0/). 